\title{
COVID-19 during pregnancy, delivery and postpartum period based on EBM
}

\author{
Patrycja Stanczyk®i), Tomasz Jachymski@, Piotr Sieroszewski® \\ $1^{\text {st }}$ Chair of Gynecology and Obstetrics, Medical University of Lodz, Poland
}

\begin{abstract}
The pandemic caused by the severe acute respiratory syndrome coronavirus 2 (SARS-CoV-2) has become the reason of the global health crisis. Since the first case of diagnosed COVID-19 pneumonia was reported in Wuhan, Hubei Province, China, in December 2019, the infection has spread rapidly to all over the world.

The knowledge gained from previous human coronavirus infection outbreaks suggests that pregnant women and their foetuses represent a high-risk population during infectious disease epidemics.

Moreover, a pregnancy, due to the physiological changes involving immune and cardiopulmonary systems, is a state predisposing women to respiratory complications of viral infection.

The constantly increasing number of publications regarding the course of COVID-19 infection in pregnant women has been published, however, the available data remains limited and many questions remain unanswered. The aim of this review was to summarize the literature data and adjusted to current recommendations regarding pregnancy care, delivery and postpartum period.

An extremely important issue is the need to register all the cases of COVID-19 affected women and the course of these pregnancies to local, regional, or international registries, which will be helpful to answer many clinical and scientific questions and to create guidelines ensuring an adequate level of care for women affected by COVID-19 infection during pregnancy, delivery and during postpartum period, as well as their newborns.
\end{abstract}

Key words: COVID-19; SARS-CoV-2; coronavirus; pandemic; pregnancy; respiratory distress syndrome

Ginekologia Polska 2020; 91, 7: 417-423

\section{INTRODUCTION}

Coronaviruses are a group of RNA viruses which are one of the largest RNA viruses in terms of the genome ( 30,000 nucleotides) and virus size (spherical, $80-180 \mathrm{~nm}$ in diameter). Their name derived from the glycoprotein spikes of the coronavirus double-layer nucleocapsid, which creates an image of the corona in the electron microscope [1, 2].

Until the end of the $20^{\text {th }}$ century, known coronaviruses infected people much less frequently, causing infections mainly in animals, being an etiological factor in the incidence of birds, cats, dogs, pigs, mice, horses, whales, camels and bats [3].

Human Coronavirus ( $\mathrm{HCoV})$, known as aetiological factors of about $15-30 \%$ of mild, seasonal infections of the lower and upper respiratory tract, can also cause gastrointestinal and rarely found nervous system infections. Thus far, eight human-infecting coronaviruses are known: HCoV-229E, HCoV-OC43, HCoV-NL63, HCoV-HKU1,
HCoV-SARS, HCoV-MERS and SARS-CoV-2, the last five of which were described in the $21^{\text {st }}$ century $[4,5]$. Coronaviruses have one of the largest genomes among RNA viruses, which, combined with the high variability characteristic of RNA viruses, leads to the accumulation of genome sequence changes, which can result in the formation of different virus variants and a change in cellular tropism [6].

The first reports of human coronaviruses originated during the 1960s when two pathogens - HCoV-229E and $\mathrm{HCoV}-\mathrm{OC} 43$ - were isolated and described - causing respiratory diseases in humans [7]. For over 40 years they were the only representatives of the Coronaviruses with the capability of causing infections in humans. The image of human coronaviruses as relatively harmless pathogens changed with the emergence of the new highly infectious human coronavirus species in the Chinese province of Guangdong in November 2002 - the SARS-CoV virus, which causes severe acute respiratory syndrome (SARS) $[8,9]$. 
During one season, the virus spread to 37 countries, 8273 cases of infection were found, of which 775 were fatal [10]. The MERS-CoV virus (Middle East Respiratory Syndrome Coronavirus) was first isolated in a patient from Saudi Arabia in 2012 [3]. By July 2017, 2040 infections were recorded, of which 712 were fatal [11].

The first cases of COVID-19 were recorded in the city of Wuhan with a population of over 11 million, the capital of the Hubei Province (60 million inhabitants) in Central China, at the beginning of December 2019. The location, transport and logistics, conducive to migration of infected people, have enabled the extremely rapid spread of the virus across all continents [12].

According to ECDC (European Center for Disease Prevention and (ontrol) data, between December 31, 2019, and July 9,2020 , there were 12017118 cases of infection found, of which 5496276 were fatal [13].

Comparison of genetic materials of coronavirus known to date indicates that SARS-CoV-2 is most likely a mutated bat-borne coronavirus that is often the asymptomatic carrier of many coronavirus species [14]. SARS-CoV-2 coronavirus has obtained human-to-human transmission ability through mutations. Its genome is $80 \%$ identical to the genome of the SARS-CoV-1 virus and 50\% identical to the MERS-CoV [15].

Particular coronaviruses use different cell receptors, which was used to divide them into three groups - depending on which receptor they recognize. The viruses belonging to group 2, which includes the SARS CoV virus, use human angiotensin 2 convertase [4]. HACE2 receptors are found on cells of many human tissues, but the strongest expression of these receptors occurs in the lungs and kidneys [16]. Protein molecules are located in the outer shell of the SARS-CoV-2 virus, which specifically binds to angiotensin 2 converting enzyme present on the surface of the airway epithelial cells and alveoli. The number of ACE2 receptors is characterized by individual variability - there are 3.5 times fewer receptors in alveoli in women than in men, which may explain the differences in COVID-19 in both sexes $[17,18]$.

Transmission of SARS-CoV-2 virus between people is mainly via a droplet route through a carrier, which is an aerosol and microdroplets emitted from the respiratory tract of an infected person containing replicated viruses. Infection can also occur through contact with the patient's secretions, the virus is not absorbed through the skin, but it can be involuntarily transmitted to the mucosa or conjunctiva. The presence of SARS-CoV-2 RNA in feces, urine and - in the case of severe infections - the blood of patients [19]. Although isolation of live virus is possible from faeces in some patients, according to a joint WHO-China report, the transmission of faecal-oral infection does not appear to be particularly important in the spread of infection [20]. Unlike other droplet-transmitted RNA viruses, such as Zika virus
(ZIKV) [21] or Ebola virus (EBOV) [22], SARS CoV 2 virus has not been found in vaginal secretions in women [23, 24].

The current, constantly deteriorating epidemiological situation worldwide, forces ongoing review of existing recommendations regarding pregnancy care, delivery and after postpartum period, considering obstetric knowledge and experience as well as an individual approach to the patient.

Some physiological changes occurring during pregnancy may increase the susceptibility of the pregnant woman to infections. Anatomical changes, such as an increase in the transverse dimension of the chest and an increase in the height at which the diaphragm is located, are factors reducing the tolerance of a pregnant woman to hypoxia [25]. Dyspnoea is a symptom affecting approximately $18 \%$ of patients with confirmed COVID-19. However, it should be remembered, that during pregnancy physiological shortness of breath is caused by increased maternal oxygen demand due to increased metabolism and oxygen consumption by the fetus, which requires differentiation from pathological dyspnoea as a manifestation of the disease [26]. Changes in lung volume and vasodilatation can lead to swelling of the mucosa and increased secretion in the upper respiratory tract. Also, changes in the immune system in relation to cellular immunity contribute to an increased susceptibility of pregnant women to infections caused by viruses [27]. Concerning the fetus and newborn, the immaturity of the immune system makes it highly prone to infections. It is particularly important to determine whether a given infectious agent can infect the fetus or newborn through vertical transmission.

Therefore, pregnant women and newborns should be assessed as potential risk groups in the current COVID-19 pandemic.

\section{MATERIAL}

The knowledge about coronavirus infection during pregnancy is limited. It is partly based on the experience of the SARS and MERS epidemics, which indicate that infection with these viruses may result in adverse clinical outcomes, including life-threatening maternal disease, which in some cases requires hospitalization, intensive care, and ventilation support. SARS and MERS infections can lead to death of pregnant women, but no specific risk factors for death during pregnancy have been identified [28].

The current review of data obtained from all published reports on pregnancy and neonatal results in women with confirmed COVID-19 is available in the Cochrane Gynecology and Fertility Group [29].

In general, although the data on pregnancy outcomes are reassuring, they should be interpreted with caution. Besides, due to the duration of the pandemic, these reports mainly relate to infections in the third trimester of preg- 
nancy. So far, no information is available on the possible impact of COVID-19 infection on the development of the first trimester of pregnancy, as well as no studies on the potential teratogenic effect on embryo-fetal development.

The collection of this information is carried out on an ongoing basis, taking the form of a wide-ranging study, e.g. the prospective ASPIRE (Assessing the Safety of Pregnancy In the Coronavirus Pandemic) cohort study is conducted in the United States, covering women who have become pregnant naturally or through assisted reproduction methods in the period from December 31, 2019, to March 1, 2020, or global reports on individual cases of pregnancies obtained as a result of assisted reproduction methods in women with confirmed infection collected by the European Society of Reproduction and Embryology [30].

In the initial period of the pandemic, the American Society for Reproductive Medicine (ASRM) and the European Society for Reproduction and Embryology (ESHRE) independently presented their opinion in which it was recommended to stop reproductive care, except in the most urgent cases $[31,32]$. Recently, thanks to effective strategies to limit the spread of COVID-19 infections in some areas, gradual resumption of full reproductive care has begun. In a document of May 29, 2020, ASRM, ESHRE and the International Federation of Fertility Societies (IFFS) jointly confirmed the opinion emphasizing the importance of continuing reproductive care during the COVID-19 pandemic. Besides, ASRM, ESHRE and IFFS work together to support patients and collect data and resources to better understand COVID-19 regarding reproduction, pregnancy, and fetal and neonatal effects [33].

The basis for current guidelines in force in Poland are the joint recommendations published on March 16, 2020, regarding the treatment of infertility of the Polish Society of Reproductive Medicine and Embryology (PTMRiE), the Fertility and Infertility Section of the Polish Society of Gynecologists and Obstetricians (PTGiP) and the National Consultant in the field of Gynecological Endocrinology and reproduction.

According to these recommendations, in the case of patients undergoing medically assisted procreation (ART) treatment, if they are at risk (i.e. who have been in places with a high incidence rate, were in contact with an infected person, are suspected of being infected COVID-19 or exhibit symptoms of inflammation of the upper respiratory tract), depending on the stage of therapy, it is recommended to stop or postpone the treatment and use the procedure of freezing oocytes or freezing embryos. If patients undergoing therapy are not at risk and are healthy, it is recommended to complete initiated infertility treatment procedures with possible consideration of deferment of the transfer. When using immunomodulatory therapies, if for any reason they are used in addition to infertility treatment, it is recommended to stop them, because in the period of the epidemic threat it can be a serious threat to health and life. Pregnant women and patients undergoing treatment or planning treatment should strictly avoid contact with infected people, those who are undergoing quarantine and avoid places where the risk of infection is increased.

All patients trying to conceive, those who are planning infertility treatment and those who are undergoing treatment, even if they do not meet the diagnostic criteria for COVID-19 infection, are advised to take precautions during therapy or postpone the treatment (limited to freezing of oocytes or embryos) [34].

Therearecurrentlyno data that would indicatean increased risk of miscarriage in patients infected with SARS CoV-2. Studies in early pregnancy in women infected with SARS-CoV and MERS-CoV also showed no significant association between infection and an increased risk of miscarriage [35].

There have been several systematic reviews in recent weeks regarding the course of COVID-19 infection in pregnant women. Zaigham et al. in a systematic review of 18 papers refer to 108 pregnant women hospitalized mainly in China, as well as from the USA, Sweden, Korea and Honduras, in whom COVID-19 infection was confirmed by PCR between December 2019 and April 2020. The average age of infected pregnant women was 29-32 years, most of the infections occurred in the third trimester. Only 22 women were in early pregnancy and were discharged without significant complications, with preserved pregnancy. Additional risk factors were found in pregnant women like chronic diseases or pregnancy complications, such as gestational diabetes, hypertension, preeclampsia and hypothyroidism [36]. Regarding the clinical course of COVID-19 infection, in most cases, the disease usually begins with fever, fatigue and dry cough. The less common symptoms include shortness of breath and tachypnoea, rhinitis, sore throat, hemoptysis, shortness of breath, headaches, and more rarely diarrhoea. According to the literature data, about $20 \%$ of patients have symptoms of interstitial pneumonia causing respiratory failure of varying severity, which requires mechanical ventilation in $3 \%$ of cases [15].

Symptoms in pregnant women may be nonspecific, besides, the asymptomatic course is often observed. Zaigham et al. report that fever (68\%) and dry cough (34\%) dominated among the clinical symptoms presented by pregnant women at the time of admission to the hospital. Fatigue (13\%), shortness of breath (12\%) and diarrhoea (6\%) were less frequently observed symptoms. Additional laboratory tests showed lymphocytopenia (59\%) and increased levels of C-reactive protein (70\%). As far as the pregnancy outcome is concerned $92 \%$ of women gave birth by caesarean section, 7 out of 85 women had a vaginal birth. There were no deaths among women in this group [36]. 
In the group of 43 pregnant women described by Breslin et al. with confirmed infection, 29 (67.4\%) manifested symptoms of infection while admitted to the hospital. Dry cough (65\%), fever (48\%) and myalgia (38\%) were the most common among the reported symptoms. Less common symptoms included: headache $(27 \%)$, shortness of breath (24\%), and chest pain (17\%). Twenty-five women in this group (86.2\%) did not require oxygen therapy or additional treatment and were discharged home in a stable condition. In the remaining four patients, the condition deteriorated, which was manifested by fever and increased respiratory effort, and the need for respiratory support and additional treatment. In one pregnant woman, an X-ray examination confirmed severe interstitial pneumonia and required admission to the ICU. The remaining 14 women did not present signs of infection at admission, 12 of them remained asymptomatic throughout the infection [37]. Breslin et al. noted in this group 2 cases of patients requiring hospitalization in ICU conditions. These women were found to have comorbidities and/or pregnancy complications such as pre-existing type 2 diabetes, poorly controlled gestational diabetes, gestational hypertension, gestational cholestasis, asthma, and BMI above $35 \mathrm{~kg} / \mathrm{m}^{2}$. Both patients delivered by caesarean section due to failure of progress or failed induction of delivery. In one patient, the surgery was complicated by uterine atony and perinatal haemorrhage. A second patient experienced respiratory failure 25 hours after cesarean section. On the fifth day after surgery, she still required oxygen therapy, and she developed acute obstetric kidney injury [38].

The effect of COVID-19 infection did not demonstrate as a cause of foetal growth restriction [39]. The presence of placental pathologies found after delivery of women infected with SARS [40] has not been confirmed.

Regarding infant mortality, Zhu et al. reported one case of neonatal death and six cases of neonates requiring admission to the Neonatal Intensive Care Unit. A symptom that occurred in all the described newborns was shortness of breath. Also, some of them had a fever, thrombocytopenia, liver dysfunction, tachycardia and vomiting. The neonatal death case concerned a premature newborn born at the 34th week of pregnancy, the result of a throat swab for SARS-CoV 2 performed on the ninth day of life was negative. As factors that contributed to the death of a newborn, Zhu et al. recognize the immaturity of the neonate's immune system with accompanying high viremia in the mother [41]. 1 case of intrauterine fetal death has been reported.

Currently, there is no literature data available confirming the possibility of intrauterine vertical transmission. The most reliable evidence for intrauterine vertical transmission would be confirmation of SARS-CoV-2 replication in fetal lungs, which is technically not possible. Intrauterine viral infection is determined based on confirmation of the presence of the virus in samples of the placenta, amniotic fluid, umbilical cord blood and newborn throat swab. To avoid contamination of samples, they should be taken immediately after delivery using aseptic technique. The first study, which examined the possibility of intrauterine transmission of COVID-19 infection, was conducted by identification of SARS-CoV-2 in samples of amniotic fluid, umbilical cord blood and neonatal throat swabs using quantitative reverse transcriptase-polymerase ( $\mathrm{qRT}-\mathrm{PCR}$ ) chain reaction in six pregnant women with confirmed COVID-19 infection and mild to moderate symptoms. Negative qRT-PCR results for SARS-CoV 2 RNA were recorded in all samples of the placenta, amniotic fluid, umbilical cord blood and nasopharyngeal swabs, indicating that there was no intrauterine fetal infection in the third trimester of pregnancy [42].

Lei et al. [43] in a study of four pregnancies in which maternal COVID-19 infection was confirmed, also showed no evidence of the intrauterine vertical transmission in the third trimester.

In March this year, two reports were published describing three cases of newborns with elevated IgM anti-SARS-CoV-2 titers in serological tests, even though the results of repeated nasopharynx swabs in qRT-PCR were negative $[44,45]$. The reason for this could be, however, the susceptibility of lgM tests to false positive and false negative results, as well as the possible cross-reactions [46].

So far, only one case of positive qRT-PCR has been described in both mother and newborn [47]. The pregnant woman was admitted to the hospital with a fever at 40 weeks of pregnancy, the nasopharyngeal swab for SARS-CoV-2 qRT-PCR test was positive, thoracic computerized tomography scan showed ground-glass opacities indicating bilateral interstitial pneumonia. A newborn was born by caesarean section in good condition. During the operation, the woman wore an N95 mask, the newborn had no contact with her after birth and was admitted to the Neonatology Department for observation. Despite the stable clinical status, the newborn had lymphocytopenia, liver dysfunction and elevated creatine kinase. A nasopharyngeal swab was taken from the newborn 36 hours after birth and came back positive. QRT-PCR results of umbilical cord blood and placenta samples were negative, but the possible transmission of SARS-CoV-2 from mother to child cannot be excluded. Both mother and child recovered and were discharged without any complications in good general condition [36].

Concerning ultrasound diagnostics during pregnancy, the Ultrasound Section of the Polish Society of Gynecologists and Obstetricians issued recommendations to minimize the risk of SARS-CoV-2 transmission. These recommendations include pre-selection of patients allowing 
ultrasound examinations only for asymptomatic patients with a negative history.

In the case of patients with a negative epidemiological history, no fever, but with cold symptoms, it is advisable to postpone the examination until the symptoms resolve. Pregnant women with a positive interview should be quarantined for 14 days and postpone the ultrasound screening examinations. In case of emergency, hospital care in a dedicated hospital is recommended. Pregnant women with diagnosed COVID-19 or with a positive SARS-CoV-2 test should have ultrasound examinations performed only in case of justified medical indications in COVID-19 dedicated hospitals. In suspected or confirmed cases of COVID-19 infection, ultrasound examination of fetal growth, amniotic fluid and umbilical artery blood flow should be performed when clinically necessary. Depending on the situation, these parameters should be monitored every $2-4$ weeks. If COVID-19 infection is confirmed during the first or early second trimester of pregnancy, an ultrasound examination should be performed between 18 and 24 weeks of pregnancy. In the case of outpatients, the first, second and third trimester scans should be performed, the remaining examinations should be postponed. Although the presence of virus RNA in the vaginal secretion has not been confirmed, due to the increased risk of virus transmission during a vaginal examination, the number of procedures performed with the vaginal transducer should be reduced, it is recommended to perform cervical length measurement by transabdominal examination [48].

According to the RCOG (Royal College of Obstetricians and Gynaecologists) recommendations from 19 June 2020 regarding the method of delivery in women with confirmed COVID-19 infection, there is no evidence indicating a specific way of delivery and it should be determined based on the obstetric situation and fetal well-being [49].

According to current recommendations of the National Consultant of Obstetrics and Gynecology, National Consultant of Neonatology, National Consultant of Perinatology, Polish Society of Gynecologists and Obstetricians, Polish Society of Neonatology and the Polish Society of Perinatal Medicine, in the case of confirmed COVID-19 infection, caesarean section for epidemiological indications should be considered, except in situations where labor is advanced when neonate will be delivered within a short time. This recommendation aims to minimize the likelihood of infection of the newborn with a virus that may be found in the birth canal. The potential exposure to maternal excretions during caesarean section is incomparably lower compared to the vaginal delivery. Also, due to the potentially shorter duration of caesarean section compared to vaginal birth, the risk of intrauterine infection, especially after rupture of amniotic membranes and perinatal infection, is significantly reduced. The above recommendations also consider the risk of transmission to the medical personnel and aim to reduce the exposure time [50].

In case of pregnant women who underwent COVID-19 without requiring admission to hospital, ended their isolation and are considered convalescents, there is no indication to modify the prior plans for the delivery [49].

In the case of anesthesia, there are no contraindications for the use of epidural or spinal anesthesia in the case of COVID-19 infection. If general anesthesia is required during cesarean section, intubation is an infectious respiratory aerosol-generating procedure, which significantly increases the risk of medical personnel infection during surgery. However, there is no evidence indicating that aerosol particles would be generated by the inhalation of a mixture of nitrous oxide and oxygen (Entonox) [51].

There are discrepancies between the recommendations for umbilical cord clamping. Chinese guidelines, to reduce the risk of vertical transmission and to isolate the newborn from the infected mother as soon as possible, do not recommend delayed umbilical cord clamping [52]. The ISUOG (International Society of Ultrasound in Obstetrics and Gynecology) guidelines do not recommend umbilical cord clamping delay [53]. The RCOG guidelines take a different opinion, indicating that delayed umbilical cord clamping should be practiced, arguing that there is no significant increase in the risk of the infection transmission in the event of an additional minute of placental transfusion [49].

Regarding breastfeeding, Martins-Filho et al. reviewed the eight available publications regarding breastfeeding of 24 symptomatic women infected with COVID-19. Biological samples were taken immediately after delivery. Nasopharyngeal swabs in newborns and placental tissue samples showed negative results for the presence of SARS-CoV-2 in qRT-PCR. Also, all breastmilk samples of infected mothers were negative. However, given the small number of cases, these reports should be treated with extreme caution, because an additional significant risk factor for the infection of the newborn during breast-feeding is close contact between the woman and the child [54].

Due to the limited data, assessment of the risk of the transmission during lactation and determination whether standard pasteurization effectively eliminates the virus is not possible. In the cases of women suspected of infection, breastmilk should be drawn, pasteurized and, while following aseptic principles, can then be given to the newborn. Breastmilk of women with confirmed infection should be utilized. However, lactation should not be suppressed in infected women if there is no medical indication so that when there is no risk of infection, they can feed naturally.

As far as the latest guidelines and the experts' statement on COVID-19 infection is concerned, newborns should be 
isolated from their mothers who have been infected until the risk of the infection stops [50]. There is no data available to determine the specific length of isolation and decisions about its end should be made individually as a result of discussions between the team of obstetricians and neonatologists [55].

RCOG in consultation with RCPCH (Royal College of Pediatrics and Child Health) advises against the routine separation of newborns from mothers with COVID-19 infection [49].

According to the Centers for Disease Control and Prevention $(C D C)$, temporary separation of the newborn from the mother with suspected or confirmed COVID-19 should be considered to reduce the risk of transmission. The risks and benefits of temporary separation of mother and newborn should be discussed with the patient. Decisions regarding temporary separation should be made according to the wishes of the mother. If the mother decides to temporarily separate, to reduce the risk of spreading the virus and would like to breast-feed, she should obtain the breast milk with a breast pump. The obtained breast milk can be given to a newborn baby by a healthy caregiver.

If a mother with suspected or confirmed COVID-19 does not decide to be temporarily separated from the neonate in hospital, she should take precautions to avoid spreading the virus to the newborn, including proper hygiene and disinfection, keeping the right distance, using barrier protection equipment, using physical barriers, e.g. an incubator [56].

\section{DISCUSSION}

Pregnant women should use standard precautions, with emphasis on personal hygiene, frequent hand sanitizing, wearing face masks, and respect social distancing and isolation.

Emphasis should be placed on routine screening for COVID-19 infection in pregnant women. Studies carried out so far show that infection in this group is usually asymptomatic or mildly symptomatic and the screening strategy allows detection of infection cases in pregnant women. Although some of the asymptomatic pregnant women developed symptoms, the severity of the disease in pregnant women appears to be similar to the general population (mild - 86\%; severe - 9.3\%, critical - 4.7\%).

Thanks to the strategy of universal screening of pregnant women, it is possible to identify a subgroup of asymptomatic women at the beginning or throughout the infection period, who have limited access to the tests. These women are currently an under-represented group in population studies [57].

Despite the constantly increasing number of publications on COVID-19 infection in pregnancy, we still do not have sufficient data to be able to draw unequivocal conclusions about the course of infection in pregnant women, effects on foetal development, maternal and neonatal outcomes, as well as vertical transmission.

Therefore, an immensely important issue is the need for systematic reporting of data on women affected by COVID-19 and the course of these pregnancies, considering the maternal and neonatal outcomes [26]. Over time, this will help to create the recommendations and guidelines that will ensure an adequate level of care for a patient affected by COVID-19 infection during pregnancy, delivery and postpartum period as well as improvement of the medical personnel protection.

\section{Conflict of interest}

None of the authors have direct or indirect conflict interest associated with publishing the article.

\section{REFERENCES}

1. Pyrć K. Ludzkie koronawirusy. Postępy Nauk Medycznych. 2015; XXVIII(4B).

2. van der Hoek L, Pyrc K, Berkhout B. Human coronavirus NL63, a new respiratory virus. FEMS Microbiol Rev. 2006; 30(5): 760-773, doi: 10.1111/j. 1574-6976.2006.00032.x, indexed in Pubmed: 16911043.

3. Zaki AM, van Boheemen S, Bestebroer TM, et al. Isolation of a novel coronavirus from a man with pneumonia in Saudi Arabia. NEngl J Med. 2012;367(19): 1814-1820, doi: 10.1056/NEJMoa1211721, indexed in Pubmed: 23075143.

4. Abramczuk E, et al. Niepandemiczne koronawirusy człowieka - charakterystyka i diagnostyka. POST. MIKROBIOL. 2017; 56(2): 205-213.

5. Vijaykrishna D, Smith GJD, Zhang JX, et al. Evolutionary insights into the ecology of coronaviruses. J Virol. 2007; 81(8): 4012-4020, doi: 10.1128/JVI.02605-06, indexed in Pubmed: 17267506.

6. Vabret A, Dina J, Brison E, et al. [Human coronaviruses]. Pathol Biol (Paris). 2009; 57(2): 149-160, doi: 10.1016/j.patbio.2008.02.018, indexed in Pubmed: 18456429.

7. Hamre D, Procknow JJ. A new virus isolated from the human respiratory tract. Proc Soc Exp Biol Med. 1966; 121(1): 190-193, doi: 10.3181/00379727-121-30734, indexed in Pubmed: 4285768.

8. KsiazekTG, Erdman D, Goldsmith CS, et al. SARS Working Group. A novel coronavirus associated with severe acute respiratory syndrome. N Engl J Med. 2003;348(20): 1953-1966, doi: 10.1056/NEJMoa030781, indexed in Pubmed: 12690092

9. Rota PA, Oberste MS, Monroe SS, et al. Characterization of a novel coronavirus associated with severe acute respiratory syndrome. Science. 2003; 300(5624): 1394-1399, doi: 10.1126/science.1085952, indexed in Pubmed: 12730500.

10. Cheng VCC, Chan JFW, To KKW, et al. Clinical management and infection control of SARS: lessons learned. Antiviral Res. 2013; 100(2): 407-419, doi: 10.1016/j.antiviral.2013.08.016, indexed in Pubmed: 23994190.

11. Plipat $T$, Buathong $R$, Wacharapluesadee $S$, et al. Imported case of Middle East respiratory syndrome coronavirus (MERS-CoV) infection from Oman to Thailand, June 2015. Euro Surveill. 2017; 22(33), doi: 10.2807/1560-7917.ES.2017.22.33.30598, indexed in Pubmed: 28840828.

12. Bogoch II, Watts A, Thomas-Bachli A, et al. Pneumonia of unknown aetiology in Wuhan, China: potential for international spread via commercial air travel. J Travel Med. 2020; 27(2), doi: 10.1093/jtm/taaa008, indexed in Pubmed: 31943059.

13. https://www.ecdc.europa.eu/en/geographical-distribution-2019-ncov-cases.

14. Sun Z, Thilakavathy K, Kumar SS, et al. Potential Factors Influencing Repeated SARS Outbreaks in China. Int J Environ Res Public Health. 2020; 17(5), doi: 10.3390/ijerph17051633, indexed in Pubmed: 32138266.

15. Rothan HA, Byrareddy SN. The epidemiology and pathogenesis of coronavirus disease (COVID-19) outbreak. J Autoimmun. 2020; 109: 102433, doi: 10.1016/j.jaut.2020.102433, indexed in Pubmed: 32113704.

16. Xiao F, Hiremath $S$, Knoll G, et al. Increased urinary angiotensin-converting enzyme 2 in renal transplant patients with diabetes. PLoS One. 2012; 7(5): e37649, doi: 10.1371/journal.pone.0037649, indexed in Pubmed: 22629438. 
17. Sun $P, L u X, X u C$, et al. Understanding of COVID-19 based on current evidence. J Med Virol. 2020; 92(6): 548-551, doi: 10.1002/jmv.25722, indexed in Pubmed: 32096567.

18. Karlberg J, Chong DSY, Lai WYY. Do men have a higher case fatality rate of severe acute respiratory syndrome than women do? Am J Epidemiol. 2004; 159(3): 229-231, doi: 10.1093/aje/kwh056, indexed in Pubmed: 14742282.

19. Peng L, Liu J, XuW et al. SARS-CoV-2 can be detected in urine, blood, anal swabs, and oropharyngeal swabs specimens. J Med Virol. 2020 [Epub ahead of print], doi: 10.1002/jmv.25936, indexed in Pubmed: 32330305.

20. Report of the WHO-China Joint Mission on Coronavirus Disease 2019 (COVID-19), 16-24 February 2020.

21. Prisant $\mathrm{N}$, Bujan $\mathrm{L}$, Benichou $\mathrm{H}$, et al. Zika virus in the female genital tract. Lancet Infect Dis. 2016; 16(9): 1000-1001, doi: 10.1016/S14733099(16)30193-1, indexed in Pubmed: 27427201.

22. Rodriguez LL, De Roo A, Guimard Y, et al. Persistence and genetic stability of Ebola virus during the outbreak in Kikwit, Democratic Republic of the Congo, 1995. J Infect Dis. 1999; 179 Suppl 1: S170-S176, doi: 10.1086/514291, indexed in Pubmed: 9988181.

23. Qiu L, Liu X, Xiao M, et al. SARS-CoV-2 is not detectable in the vaginal fluid of women with severe COVID-19 infection. Clin Infect Dis. 2020 [Epub ahead of print], doi: 10.1093/cid/ciaa375, indexed in Pubmed: 32241022.

24. Cui $\mathrm{P}, \mathrm{Chen} \mathrm{Z}$, Wang $\mathrm{T}$, et al. Clinical features and sexual transmission potential of SARS-CoV-2 infected female patients: a descriptive study in Wuhan, China. , doi: 10.1101/2020.02.26.20028225.

25. O'Day MP. Cardio-respiratory physiological adaptation of pregnancy. Semin Perinatol. 1997; 21(4): 268-275, doi: 10.1016/s01460005(97)80069-9, indexed in Pubmed: 9298715.

26. Dashraath P, Wong JL, Lim MX, et al. Coronavirus disease 2019 (COVID-19) pandemic and pregnancy. Am J Obstet Gynecol. 2020; 222(6): 521-531, doi: 10.1016/j.ajog.2020.03.021, indexed in Pubmed: 32217113.

27. Nelson-Piercy C. Respiratory disease. In: Nelson-Piercy C. ed. Handbook of Obstetric Medicine. CRC Press, Boca Raton, FL 2015: 63-84.

28. Schwartz DA, Graham AL. Potential Maternal and Infant Outcomes from (Wuhan) Coronavirus 2019-nCoV Infecting Pregnant Women: Lessons from SARS, MERS, and Other Human Coronavirus Infections. Viruses. 2020; 12(2), doi: 10.3390/v12020194, indexed in Pubmed: 32050635.

29. https://cgf.cochrane.org/news/covid-19-koronawirus-chorobapłodność-i-ciąża.

30. https://nl.surveymonkey.com/r/COVID19ART.

31. https://www.asrm.org/globalassets/asrm/asrm-content/news-and-publications/covid-19/covidtaskforce.pdf.

32. Assisted reproduction and COVID-19. A statement from ESHRE for phase 1 - Guidance on fertility services during pandemic. https://www.eshre. eu/Press-Room/ESHRE-News\#CoronaStatement27feb.

33. https://www.asrm.org/globalassets/asrm/asrm-content/news-and-publications/covid-19/asrm-eshre-iffs-joint-statement.pdf.

34. http://www.ptgin.pl/koronawirus-covid-19-stanowisko-w-kwestii-leczenia-nieplodnosci.

35. Zhang Jp, Wang Yh, Chen Ln, et al. [Clinical analysis of pregnancy in second and third trimesters complicated severe acute respiratory syndrome]. Zhonghua Fu Chan Ke Za Zhi. 2003; 38(8): 516-520, indexed in Pubmed: 14521763.

36. Zaigham M, Andersson O. Maternal and perinatal outcomes with COVID-19: A systematic review of 108 pregnancies. Acta Obstet Gynecol Scand. 2020; 99(7): 823-829, doi: 10.1111/aogs.13867, indexed in Pubmed: 32259279.

37. Breslin N, Baptiste C, Gyamfi-Bannerman C, et al. COVID-19 infection among asymptomatic and symptomatic pregnant women: Two weeks of confirmed presentations to an affiliated pair of New York City hospitals. Am J Obstet Gynecol MFM. 2020 [Epub ahead of print]: 100118, doi: 10.1016/j.ajogmf.2020.100118, indexed in Pubmed: 32292903.

38. Breslin N, Baptiste C, Miller R, et al. Coronavirus disease 2019 in pregnancy: early lessons. Am J Obstet Gynecol MFM. 2020; 2(2): 100111, doi: 10.1016/j.ajogmf.2020.100111.
39. Mullins E, Evans D, Viner RM, et al. Coronavirus in pregnancy and delivery: rapid review. Ultrasound Obstet Gynecol. 2020; 55(5): 586-592, doi: 10.1002/uog.22014, indexed in Pubmed: 32180292.

40. Ng WF, Wong SF, Lam A, et al. The placentas of patients with severe acute respiratory syndrome: a pathophysiological evaluation. Pathology. 2006; 38(3): 210-218, doi: 10.1080/00313020600696280, indexed in Pubmed: 16753741.

41. Zhu H, Wang $L$, Fang $C$, et al. Clinical analysis of 10 neonates born to mothers with 2019-nCoV pneumonia. Transl Pediatr. 2020; 9(1): 51-60, doi: 10.21037/tp.2020.02.06, indexed in Pubmed: 32154135.

42. Chen $\mathrm{H}$, Guo J, Wang $\mathrm{C}$, et al. Clinical characteristics and intrauterine vertical transmission potential of COVID-19 infection in nine pregnant women: a retrospective review of medical records. Lancet. 2020; 395(10226): 809-815, doi: 10.1016/S0140-6736(20)30360-3, indexed in Pubmed: 32151335.

43. Lei D, Wang C, Li C, et al. Clinical characteristics of COVID-19 in pregnancy: analysis of nine cases. Chin J Perinat Med. 2020; 23(03): 225-231.

44. Zeng $\mathrm{H}, \mathrm{Xu} \mathrm{C}$, Fan J, et al. Antibodies in Infants Born to Mothers With COVID-19 Pneumonia. JAMA. 2020 [Epub ahead of print], doi: 10.1001/jama.2020.4861, indexed in Pubmed: 32215589.

45. Dong L, Tian J, He S, et al. Possible Vertical Transmission of SARS-CoV-2 From an Infected Mother to Her Newborn. JAMA. 2020 [Epub ahead of print], doi: 10.1001/jama.2020.4621, indexed in Pubmed: 32215581.

46. Kimberlin DW, Stagno S. Can SARS-CoV-2 Infection Be Acquired In Utero?: More Definitive Evidence Is Needed. JAMA. 2020 [Epub ahead of print], doi: 10.1001/jama.2020.4868, indexed in Pubmed: 32215579.

47. Wang S, Guo L, Chen L, et al. A case report of neonatal COVID-19 infection in China. Clin Infect Dis. 2020 [Epub ahead of print], doi: 10.1093/cid/ciaa225, indexed in Pubmed: 32161941.

48. Zalecenie Sekcji Ultrasonografii PTGIP w zakresie badań USG w sytuacji epidemiologicznej- COVID-19. http://www.ptgin.pl/zalecenie-sekcji-ultrasonografii-ptgip-w-zakresie-badan-usg-w-sytuacji-epidemiologicznej-covid-19.

49. Coronavirus (COVID - 19) Infection in Pregnancy. RCOG. https://www rcog.org.uk/globalassets/documents/guidelines/2020-06-18-coronavirus-covid-19-infection-in-pregnancy.pdf.

50. Najnowsze wytyczne i stanowisko ekspertów w sprawie zakażenia wirusem COVID-19. http://ptmp.edu.pl/najnowsze-wytyczne-i-stanowisko-ekspertow-w-sprawie-zakazenia-wirusem-covid-19.

51. Morau E, Bouvet L, Keita H, et al. CAROWorking Group. Anaesthesia and intensive care in obstetrics during the COVID-19 pandemic. Anaesth Crit Care Pain Med. 2020; 39(3): 345-349, doi: 10.1016/j.accpm.2020.05.006, indexed in Pubmed: 32405520.

52. Wang L, Shi Y, Xiao T, et al. Chinese expert consensus on the perinatal and neonatal management for the prevention and control of the 2019 novel coronavirus infection (First edition). Annals of Translational Medicine. 2020; 8(3): 47-47, doi: 10.21037/atm.2020.02.20.

53. Poon LC, Yang H, Lee J, et al. ISUOG Interim Guidance on 2019 nove coronavirus infection during pregnancy and puerperium: information for healthcare professionals. Ultrasound Obstet Gynecol. 2020; 55(5): 700-708, doi: 10.1002/uog.22013.

54. Martins-Filho PR, Santos VS, Santos HP. To breastfeed or not to breastfeed? Lack of evidence on the presence of SARS-CoV-2 in breastmilk of pregnant women with COVID-19. Rev Panam Salud Publica. 2020; 44: e59, doi: 10.26633/RPSP.2020.59, indexed in Pubmed: 32454808.

55. Rasmussen SA, Smulian JC, Lednicky JA, et al. Coronavirus Disease 2019 (COVID-19) and pregnancy: what obstetricians need to know. Am J Obstet Gynecol. 2020; 222(5):415-426, doi: 10.1016/j.ajog.2020.02.017, indexed in Pubmed: 32105680.

56. https://www.cdc.gov/coronavirus/2019-ncov/need-extra-precautions/pregnancy-breastfeeding.html\#breastfeeding.

57. Guan WJ, Ni ZY, Hu Yu, et al. China Medical Treatment Expert Group for Covid-19. Clinical Characteristics of Coronavirus Disease 2019 in China. N Engl J Med. 2020; 382(18): 1708-1720, doi: 10.1056/NEJMoa2002032, indexed in Pubmed: 32109013 